\title{
To the question of action on the rods lying on elastic foundation, inertial load with a variable speed of its movement
}

\author{
Igor Ivanchenko* \\ Russian University of Transport, 127994, Moscow, Russian Federation
}

\begin{abstract}
A method for calculating the rods on elastic foundation under the inertial load action when it moves at a variable speed is proposed. Test problems about a force or load movement with variable speeds along a hinged beam and about the movement with a high-speed railway car deceleration along a track section modeled by a hinged supported beam of great length on an elastic foundation are considered. The selection of the elastic foundation material of the rail track determines the dynamics of the high-speed railway car in different modes of its movement. To construct the methodology, the previously proposed by the author of the article solutions are used: a step-by-step procedure for solving the problems of unsteady dynamics of structures and the method of "nodal accelerations" to take into account the action on structures of a moving inertial load.
\end{abstract}

\section{Introduction}

The tasks of studying the interaction of high-speed rolling stock and railway tracks remain relevant [1-9]. The described method for solving the problems of a moving load takes into account any required number of vibration modes in the rod deflection function expansion and leads to a resolving system of equations when using an unconditionally stable integration scheme with a minimum number of the unknowns, as by the method of integral equations when solving problems at a constant movement speed. As the load on the rods, concentrated forces, loads and carriages moving at a variable speed are considered.

\section{Problem statement, general formulas and test cases}

At the beginning let us turn to the solution of the classical problem of a load movement along a beam on an elastic foundation with a variable speed, and then proceed to the case of a more complex load. Further an unconditionally stable step-by-step procedure in time and the method of taking into account the action of a massless moving load on rod systems,

\footnotetext{
* Corresponding author: ivaii011@mtu-net.ru
} 
proposed earlier are used [4]. The cases of uniformly variable motion of the moving load on the rods will be considered.

The differential equation of vibrations of a beam on an elastic foundation when load $P$ moves along it and mass $M$ has the form

$$
L_{1} q^{*}(y, t)=\delta(y-s(t))\left\{P-M \frac{d^{2} q^{*}(s(t), t)}{d t^{2}}\right\}, \quad L_{1}=E J \frac{\partial^{4}}{\partial y^{4}}+\mu_{1} E J \frac{\partial^{5}}{\partial t \partial y^{4}}+\gamma \frac{\partial}{\partial t}+k()+m \frac{\partial^{2}}{\partial t^{2}}
$$

Here $E J$ defines bending stiffness of the beam, $\mu_{1}, \gamma$ are the energy dissipation factors, $k$ is the modulator of subgrade reaction, $q^{*}(y, t)$ is beam deflection, $y$ is length coordinate, $\delta(y-s(t))$ is delta function, $s(t)=s_{0}+v_{0} t+w t^{2} / 2$ defines the law of load movement along the beam, $v_{0}$ is the load speed at the entrance to the beam, $v=v_{0}+w t$ and $w$ define load speed and acceleration.

Deflection of a hinged beam at the moment $t_{j+1}$ when concentrated force moves along it,

can be written as $[4,5]$

$$
R=\delta(y-s(t))\left(P-M \ddot{q}_{1}\right)
$$

$$
\begin{gathered}
q^{*}\left(\eta_{1}, t_{j+1}\right)=\sum_{i=1}^{n} W_{i}\left(\eta_{1}\right) q_{i j+1}=\sum_{i=1}^{n} W_{i}\left(\eta_{1}\right)\left(q_{i j}+\dot{q}_{i j} \Delta t_{j}+\ddot{q}_{i j+1 / 2} \frac{\Delta t_{j}^{2}}{2}\right)= \\
=\sum_{i=1}^{n} W_{i}\left(\eta_{1}\right)\left\{\left(1-\vartheta_{i j} \widetilde{\omega}_{i}^{2}\right) q_{i j}+\left(\Delta t_{j}-\vartheta_{i j}\left(\widetilde{\mu}_{i}+\frac{\Delta t_{j}}{2} \widetilde{\omega}_{i}^{2}\right)\right) \dot{q}_{i j}+\vartheta_{i j} \int_{0}^{1} \bar{\eta}_{j+1 / 2} d \eta\right\} \\
W_{i}\left(\eta_{1}\right)=\sin \left(r_{i} \eta_{1}\right), \quad \vartheta_{i j}=\frac{\Delta t_{j}^{2}}{2}\left(1+\widetilde{\mu}_{i} \frac{\Delta t_{j}}{2}+\frac{\Delta t_{j}^{2} \widetilde{\omega}_{i}^{2}}{4}\right)^{-1}, \widetilde{\omega}_{i}=\left(\left(\frac{r_{i}}{\ell}\right)^{4} \frac{E J}{m}+\frac{k}{m}\right)^{1 / 2} \\
\widetilde{\mu}_{i}=\mu_{1}\left(\frac{r_{i}}{\ell}\right)^{4} \frac{E J}{m}+\frac{\gamma}{m}, \quad r_{i}=\pi i, \quad \eta_{1}=y / \ell, \quad \Delta t_{j}=t_{j+1}-t_{j} \\
\bar{\eta}=\tilde{\sigma} W_{i}\left(\eta_{1}\right), \quad \tilde{\sigma}=\frac{2}{m \ell}, i=1, \ldots, n, \quad j=0,1.2, \ldots .
\end{gathered}
$$

Here $q_{i j}$ defines the generalized beam coordinates, $W_{i}\left(\eta_{1}\right)$ determine fundamental functions, $\widetilde{\omega}_{i}$ is bending circular frequency, $n$ is a number of retained waveforms, $\ell$ is the beam length, $\ddot{q}_{1}$ defines the vertical acceleration of the load.

Assuming $\ddot{q}_{1 j+1 / 2}=d^{2} q^{*}(s(t), t) / d t^{2}$, at $t=t_{j+1 / 2}$, we can find the total vertical acceleration of the load in the form

$$
\ddot{q}_{1 j+1 / 2}=\left.\frac{d^{2} q^{*}(s(t), t)}{d t^{2}}\right|_{t=t_{j+1 / 2}}=\frac{\partial^{2} q^{*}}{\partial t^{2}}+2 \frac{\partial^{2} q^{*}}{\partial s \partial t} \frac{d s}{d t}+\frac{\partial^{2} q^{*}}{\partial s^{2}}\left(\frac{d s}{d t}\right)^{2}+\left.\frac{\partial q^{*}}{\partial s} \frac{d^{2} s}{d t^{2}}\right|_{t=t_{j+1 / 2}}
$$

Using (2) and (3) at the step we get $\left[t_{j}, t_{j+1}\right]$ 


$$
\begin{gathered}
\ddot{q}_{1 j+1 / 2}=\sum_{i=1}^{n}\left\{\alpha_{1 i j} \ddot{q}_{i j+1 / 2}+\alpha_{2 i j} \dot{q}_{i j}+\alpha_{3 i j} q_{i j}\right\} \\
\alpha_{1 i j}=\sin \left(r_{i} \eta_{1+1 / 2}\right)+\frac{r_{i} \Delta t_{j}}{\ell}\left[v_{0}+w t_{j+1 / 2}\right] \cos \left(r_{i} \eta_{1+1 / 2}\right)+\widetilde{d}_{i j} \frac{\Delta t^{2}}{4}, \\
\alpha_{2 i j}=\frac{2 r_{i}}{\ell}\left[v_{0}+w t_{j+1 / 2}\right] \cos \left(r_{i} \eta_{1+1 / 2}\right)+\widetilde{d}_{i j} \frac{\Delta t}{2}, \\
\alpha_{3 i j}=\widetilde{d}_{i j}, \tilde{d}_{i j}=-\left(v_{0}^{2}+2 w t_{j+1 / 2} v_{0}+w^{2} t_{j+1 / 2}^{2}\right)\left(\frac{r_{i}}{\ell}\right)^{2} \sin \left(r_{i} \eta_{1+1 / 2}\right)+w\left(\frac{r_{i}}{\ell}\right) \cos \left(r_{i} \eta_{1+1 / 2}\right) \\
\ddot{q}_{i j+1 / 2}=\left\{\begin{array}{c}
\eta_{1 j+1 / 2}=\left(v_{0} t_{j+1 / 2}+w \frac{t_{j+1 / 2}^{2}}{2}\right) / \ell, \\
\left.-\widetilde{\omega}_{i}^{2} q_{i j}-\left(\widetilde{\mu}_{i}+\frac{\Delta t_{j}}{2} \widetilde{\omega}_{i}^{2}\right) \dot{q}_{i j}+\widetilde{\sigma}_{\operatorname{b}} \sin \left(r_{i} \eta_{1+1 / 2}\right)\left(P+R_{1 j+1 / 2}\right)\right\} \frac{2 \vartheta_{i j}}{\Delta t_{j}^{2}}
\end{array}\right.
\end{gathered}
$$

From the conditions of the dynamic balance of the load we find:

$$
R_{1 j+1 / 2}=-M \ddot{q}_{1 j+1 / 2}
$$

after substituting (5) into (4), at the step $\left[t_{j}, t_{j+1}\right]$ the equation is $\ddot{q}_{1 j+1 / 2}$. The expression (2), taking into account (5), allows, taking into account the conditions of continuity at the load contact point, to calculate the initial conditions of the problem for the next integration step. Considering a beam on an elastic foundation, we use a series of test cases for a beam without an elastic foundation.

The step procedure (2) - (5) is implemented for the uniformly variable movement of the load along the beam with the parameters [1-3].

$$
\beta=\frac{P}{m \ell g}, \quad \alpha=\frac{v l}{\pi \sqrt{E J / m}}, \quad w=\frac{v^{2}}{2 \ell}\left(\left(\frac{v_{1}}{v_{0}}\right)^{2}-1\right), \quad \widetilde{\beta}=\left(\frac{k}{4 E J}\right)^{1 / 4}
$$

where $v_{0}$ and $v_{1}$ represent speed, respectively, of the load entry and exit from the beam.

Figure 1 shows the nature of the dynamic coefficient increase and decrease $Z_{y=\ell / 2}=Z_{D} / Z_{0}$ depending on the movement speed, determined by the coefficient $\alpha$ by $v_{1} / v_{0}=2 / 3$ in (6) and the ratio of the load and the beam masses in (6) at $\beta$ equal, respectively, to the values $3,2,1,0.5$ and 0.25 (refer with figure 1), while $Z_{D}$ is the greatest displacement of the load and $Z_{0}=2 \mathrm{Pl}^{3} /\left(\pi^{4} \mathrm{EJ}\right)$ defines static deflection under force in the center of the beam at $\widetilde{\beta}=0$ and $v=v_{0}$ in (6). The results obtained by different methods and presented in Fig. 1 and in [3] (p. 210), practically coincide. 


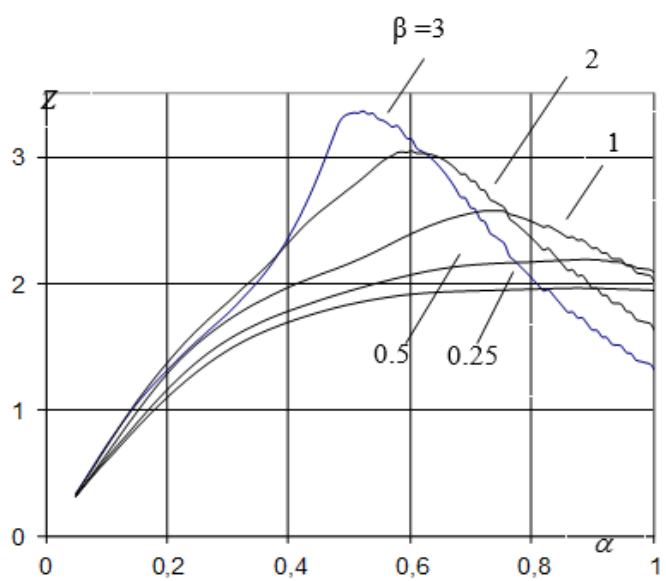

Fig. 1. The nature of the dynamic coefficient increase and decrease

For the next numerical experiment, a beam with the length $\ell=13 \mathrm{~m}$ on an elastic foundation, with the parameters corresponding to the railway track: $E=2.1 \times 10^{8} \mathrm{kN} / \mathrm{m}^{2}$; $J=0.7 \times 10^{-4} \mathrm{~m}^{4}, \quad k=3.0 \times 10^{4} \mathrm{kN} / \mathrm{m}^{2}, \quad m=0.15 \mathrm{t} / \mathrm{m}, \mu_{1}=\mu_{i}=0, \quad \widetilde{\mu}_{1}=\tilde{\mu}_{i}=0 \quad$ by $\widetilde{\beta}=0.845 \mathrm{~m}^{-1}$ are chosen.

It should be noted that the proposed method (2) - (5), for $M=0$, can be used to solve the classical problem, motion with variable speed along the concentrated force rod [1]. Figure $2(a, b)$ shows the following changes: $\left.Z\right|_{y=\ell / 2}=Z_{D} / Z_{0}$ and $\left.Z\right|_{y=\ell / 2}=Z_{D} / \widetilde{Z}_{0}$ are the dynamic coefficients for displacements $Z_{D}$ in the center of the beam with an equally variable motion of a concentrated force, respectively, with $\widetilde{\beta}=0$ (Fig. 2a) and $\widetilde{\beta}=0.845 \mathrm{~m}^{-1}$ (Fig. $2 b$ ), where $Z_{0}$ is static deflection under force in the center of the beam at $\widetilde{\beta}=0$ and $\widetilde{Z}_{0}$ by $\widetilde{\beta}=0.845 \mathrm{~m}^{-1}$ (Fig. $2 b$ ), $\left.\quad \widetilde{Z}\right|_{y=\ell / 2}=Z_{D} / \widetilde{Z}_{0}$ are the dynamic coefficients for displacements when moving the load at $\widetilde{\beta}=0$ (fig. 2c) and at $\widetilde{\beta}=0.845 \mathrm{~m}^{-1}$ (Fig. 2e), $R=P_{D} / P$ are the dynamic coefficients for the load dynamic pressure, where $P_{D}$ is pressure under load at $\widetilde{\beta}=0$ (Fig. $2 d$ ) and by $\widetilde{\beta}=0.845 \mathrm{~m}^{-1}$ (Fig. 2f) depending on the displacement of the force along the beam, determined by the segments $\xi=\pi t v / \ell$ at $\alpha=0.5$ and $\beta=1$ [1]. In all the cases considered in figure $2(a, b, c, d, e, f)$ with parameter $B=w \ell / v^{2}[1]$ the lines 1 correspond to uniformly slow motion at $w=-v_{0}^{2} / 2 \ell$, $v_{1}=0, t_{1}=2 \ell / v_{0}, B=-0.5, \quad v=v_{0}$, and the line 2 accelerated motion at $w=v_{1}^{2} / 2 \ell, \quad v_{0}=0, t_{2}=2 \ell / v_{1}, B=0.5, v=v_{1}$, where $t_{1,2}$ is the time of load movement, $\Delta t_{j}=0.0005 \mathrm{c}, n=45$ (by $\beta=0$ ) and $n=150$ (by $\beta=0.845 \mathrm{~m}^{-1}$ ) with the number of time steps equal to $\mathrm{N}=1376$. To determine the static deflection under the force of a beam on an elastic foundation, a tested technique based on the application of a step-bystep procedure was used, as well as in this article, from [4, 8], where, when solving dynamic 
problems at the first integration step, for example, equal to $\Delta t_{j}=10 \mathrm{~s}$, and zero initial conditions, the doubled static deflection is determined at the corresponding point in the structure. Thus, using the algorithm (1.2) - (1.5) for the case of the action of a suddenly applied force $P$, by $v_{0}=w=0$ and $s_{0}=6.5 \mathrm{~m}$ we have at $\beta=1$ and $\widetilde{\beta}=0.845 \mathrm{~m}^{-1}$, for the considered beam $\delta_{C T \text {., } / 2}=0.000269 \mathrm{~m}$, which practically coincides with the value $\widetilde{Z}_{0}=P /\left(8 \widetilde{\beta}^{3} E J\right)$ for deflection under force at an endless beam.

The results obtained by different methods for the force movement case and presented in Fig. $2 a$ and in [1] (p. 313), practically coincide.

(a)

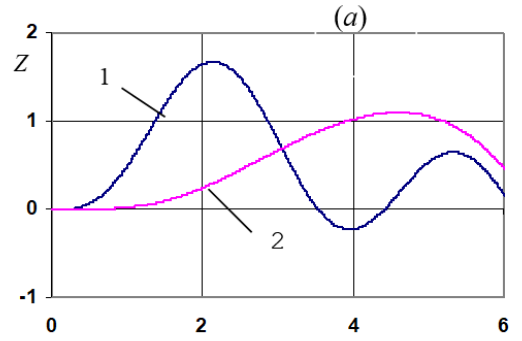

(c)

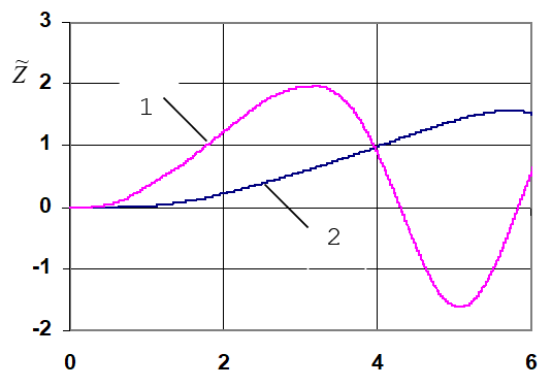

(b)

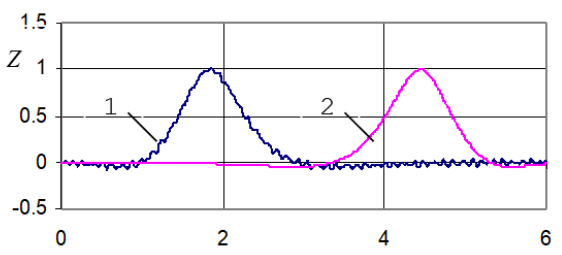

(d)

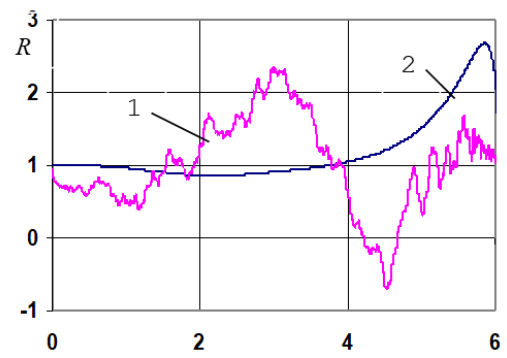

e)

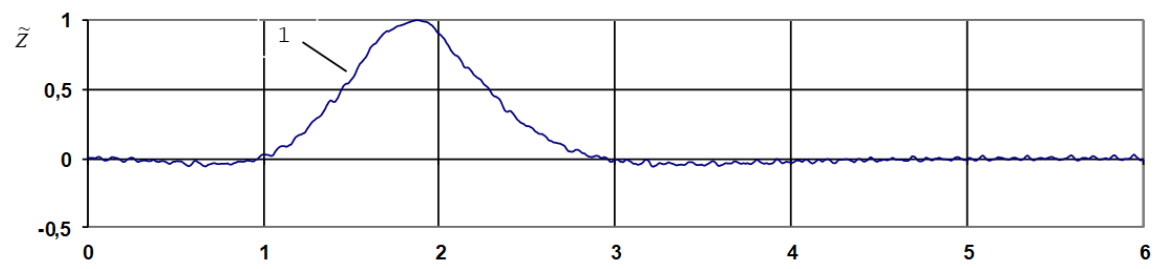

(f)

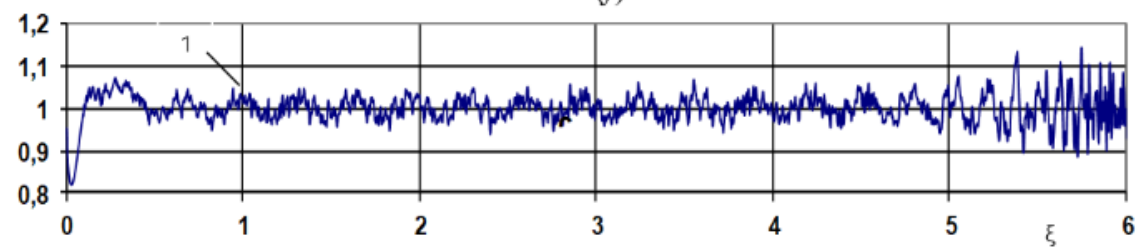

Fig. $2(a, b, c, d, e, f)$. 


\section{Method for solving the equations for the "car-track" system.}

The algorithm (2) - (4) is easily implemented in the case of movement of a system of loads along a beam on an elastic foundation. The expressions of the form (4) when moving $\tilde{N}$ cargo form a system of $\widetilde{N}$ linear, algebraic equations

$$
\widetilde{\mathbf{A}} \mathbf{R}_{k o j+1 / 2}+\mathrm{E} \ddot{\mathbf{q}}_{K j+1 / 2}=\widetilde{\mathbf{B}}
$$

Here $\widetilde{\mathbf{A}}, \widetilde{\mathbf{B}}$ are matrix and vector, $\mathrm{E}$ is an identity matrix, $\mathbf{R}_{k o}$ is the vector of dynamic additions to the static pressure of loads on the beam, $\ddot{\mathbf{q}}_{K}$ is the vector of vertical acceleration of moving loads.

Let us consider a model for studying the vertical dynamics of the experimental system "track-train" for the possibility of studying their interaction. We will further denote this system by $\left\{e_{h}, e_{h^{o}}^{\circ}\right\}$ ( Fig. $\left.3 a, 4\right)$, where $e_{h}$ is the rail-beam, on an elastic foundation, $e_{h^{\circ}}^{\circ}$ is railway car.

(a)

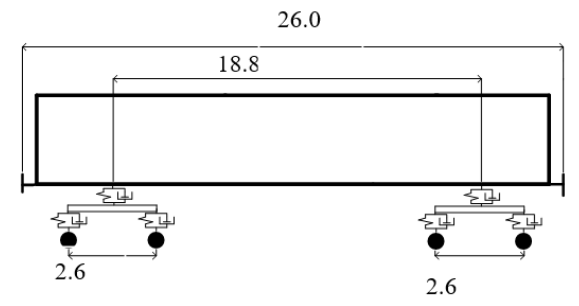

(b)

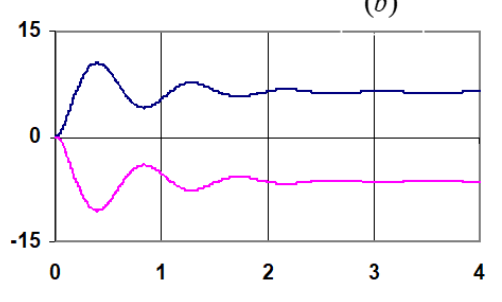

Fig. $3(a, b)$.

Let us construct a system of equations describing the vertical dynamics of the car $e_{h^{o}}^{0}$. In this case, we will assume that the initial conditions of the problem are zero, and the parameters determining the position $e_{h^{o}}^{\circ}$ in the system $O_{*} X_{*} Y_{*} Z_{*}$, moving at the car speed progressively, are counted from their values in static equilibrium, while at the moment of the braking start, a horizontal inertia force is applied to the car body, affecting the vertical dynamics of the car (the longitudinal dynamics of the composition is not considered). Then for $e_{h^{o}}^{\circ}$ we have

$$
\mathbf{M}_{*} \ddot{\overline{\mathbf{q}}}_{c}+\mathbf{C}_{*} \dot{\overline{\mathbf{q}}}+\mathbf{K}_{*} \overline{\mathbf{q}}_{c}=\overline{\mathbf{R}}_{*}, \overline{\mathbf{R}}_{*}=\Pi_{*} \mathbf{R}_{*}
$$

Here $\overline{\mathbf{q}}_{c}$ is the vector of independent generalized coordinates defining $e_{h^{o}}^{\circ}$ in the system $O_{*} X_{*} Y_{*} Z_{*}, \mathbf{M}_{*}, \mathbf{C}_{*}, \mathbf{K}_{*}$ are the matrices of masses, damping and stiffness for $e_{h^{o}}^{\circ}[8], \Pi_{*}$ is the vector connection matrix $\overline{\mathbf{R}}_{*}$ and $\mathbf{R}_{*}, \mathbf{R}_{*}$ is the vector of dynamic additions to static reactions at the points of contact $e_{h^{o}}^{\circ}$ with carriageway (with rail - beam $e_{h}$ and a churlish railroad body outside $e_{h}$ ). 
We will assume that the car braking when moving at a constant speed along the railbeam (Fig. 4) occurs at the moment $t^{*}$, when the first wheelset of the carriage reaches the middle $e_{h}$, while at the moment $t^{*}$ an inertial pair of forces is applied to the car body $M^{(\text {inert })}=M^{(c a b)} w \times L$ (refer with Fig. 4), where $M^{(c a b)}$ is wagon body weight, $L-$ is the vertical distance from the body mass center to the wheelset axle. Let us introduce into consideration the following: $\mathbf{P}_{*}$ and $\mathbf{R}_{k o}$ are vectors of static pressures of wheelsets $e_{h^{o}}^{\circ}$ on the carriageway and the corresponding dynamic additives to them, $\mathbf{q}_{c k}$ is a displacement vector of the car wheels (sub-vector $\overline{\mathbf{q}}_{c}$ ), $\mathbf{q}_{k o}$ is a vector of displacements of the carriageway at the points of contact with it of the car wheelsets. Let us select the subvectors from $\mathbf{R}_{k o}, \mathbf{R}_{*}, \mathbf{q}_{k o}, \mathbf{q}_{c k}$, respectively, $\mathbf{R}_{k o}^{\circ}, \mathbf{R}_{*}^{\circ}, \mathbf{q}_{k o}^{\circ}, \mathbf{q}_{c k}^{\circ}$, responsive at the entrance to the interaction of wheelsets $e_{h^{o}}^{\circ}$ only with $e_{h}$, where $\mathbf{R}_{k o}^{\circ}, \mathbf{R}_{*}^{\circ}$ are the dynamic additives to static wheel pressures and additives to wheel reactions,

$\mathbf{q}_{k o}^{\circ}, \mathbf{q}_{c k}^{\circ}$ are the beam and wheel displacements on the beam-rail. At any given time, the elements of the vector $\mathbf{q}_{k o}$, related to the carriageway outside $e_{h}$, remain zero, and when the car moves along $e_{h}$ the continuity conditions of displacements and velocities are met $\mathbf{q}_{k o}^{\circ}=\mathbf{q}_{c k}^{\circ}, \dot{\mathbf{q}}_{k o}^{\circ}=\dot{\mathbf{q}}_{c k}^{\circ}$ as well as the conditions of equilibrium in the moving nodes (at the points of contact of the wheels with the rail - a beam on an elastic foundation).

$$
\mathbf{R}_{k o}^{\circ}+\mathbf{R}_{*}^{\circ}=0
$$

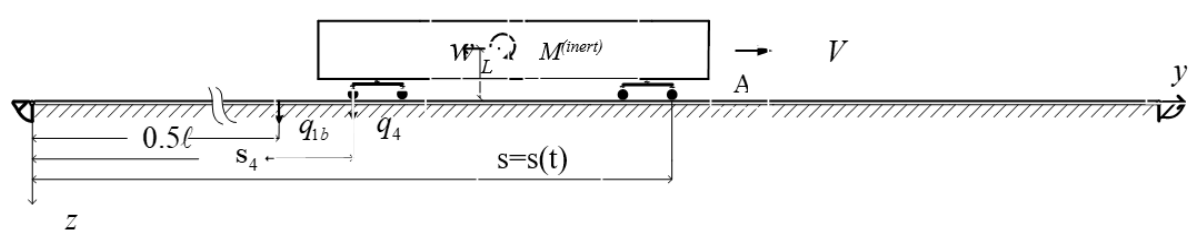

Fig. 4.

For the rail-beam $e_{h}$, along which the carriage moves, at a step $\left[t_{j}, t_{j+1}\right]$ the following equation can be written

$$
\mathbf{A} \mathbf{R}_{k 0 j+1 / 2}^{o}+\mathbf{E} \ddot{\mathbf{q}}_{k 0 j+1 / 2}^{o}=\mathbf{B}
$$

Here $\mathbf{A}$ is a matrix characterizing stiffness, dissipative and inertial characteristics $e_{h}, E$ is an identity matrix, $\mathbf{B}$ is the vector taking into account the initial conditions for $e_{h}$ in the moment $t_{j}$ and the action on the moving forces $\mathbf{P}_{*}$ and $\mathbf{R}_{k o}$ carriageway system.

Let us carry out discretization ( 8$)$ in time $(j=0,1,2 \ldots)$, using the step-by-step procedure from $[4,8]$, as a result, we have 

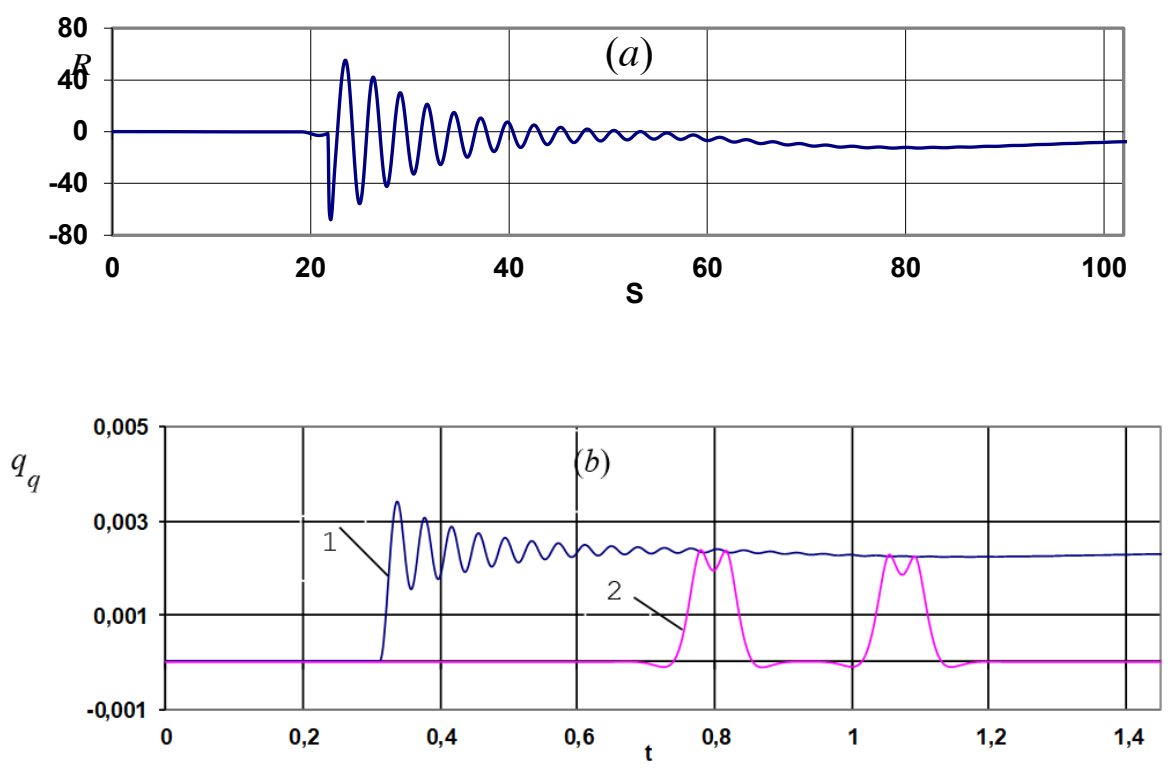

(c)

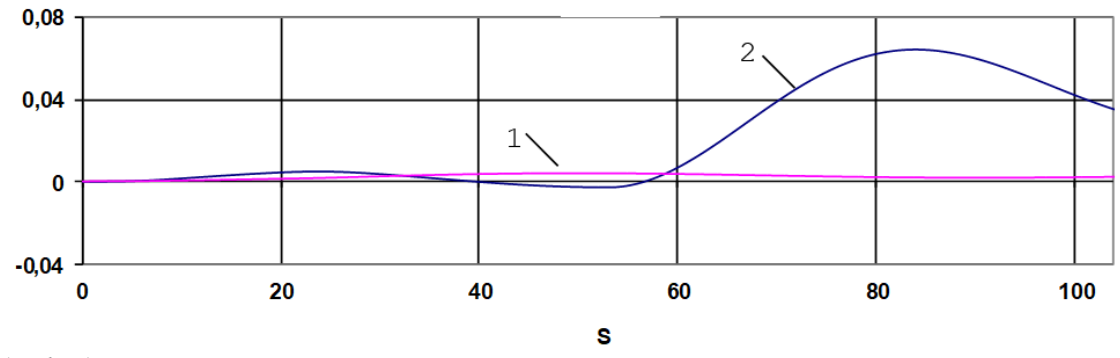

Fig. $5(a, b, c)$

$$
\ddot{\overline{\mathbf{q}}}_{c j+1 / 2}=\widetilde{\mathbf{G}}_{1} \overline{\mathbf{q}}_{c j}+\widetilde{\mathbf{G}}_{2} \dot{\mathbf{q}}_{c j}+\mathbf{A}+\boldsymbol{\Pi} \cdot \mathbf{R}^{* j+1 / 2}
$$

$$
\begin{gathered}
\mathbf{A}^{*}=\left[\mathbf{M} * \mathbf{C}_{*} \frac{\Delta t_{j}}{2}+\mathbf{K} * \frac{\Delta t_{j}^{2}}{4}\right]^{-1}, \quad \widetilde{\mathbf{G}}_{1}=-\mathbf{A}^{*} \mathbf{K}_{*}, \widetilde{\mathbf{G}}_{2}=-\mathbf{A}^{*}\left(\mathbf{C}_{*}+\mathbf{K}_{*} \frac{\Delta \boldsymbol{t}_{j}}{2}\right) \\
\overline{\mathbf{q}}_{c j+1}=\overline{\mathbf{q}}_{c j}+\dot{\overline{\mathbf{q}}}_{c j} \Delta \boldsymbol{t}_{j}+\ddot{\overline{\mathbf{q}}}_{c j+1 / 2} \frac{\Delta t_{j}^{2}}{2}, \quad \dot{\overline{\mathbf{q}}}_{c j+1}=\dot{\overline{\mathbf{q}}}_{c j}+\ddot{\overline{\mathbf{q}}}_{c j+1 / 2} \Delta \boldsymbol{t}_{j}
\end{gathered}
$$

Let us select a subsystem of equations corresponding to the sub-vector on the left-hand side $\ddot{\mathbf{q}}_{c k j+1 / 2}^{\circ}$ from (11); further we will express this subsystem with respect to the vector of dynamic additions to the static reactions of the wheels, presenting it in the form:

$$
\mathbf{R}_{* j+1 / 2}^{o}=\mathbf{W}^{o} \ddot{\mathbf{q}}_{k o j+1 / 2}^{o}+\mathbf{L}^{o}
$$


The vectors $\mathbf{R}_{k o}^{o}, \mathbf{R}_{*}^{o}$ from (10) and (13) are substituted to (9) the as a result, we have a resolving system of equations at the step $\left[t_{j}, t_{j+1}\right]$

$$
\mathbf{D} \ddot{\mathbf{q}}_{k o j+1 / 2}^{o}=\mathbf{D}^{o}
$$

$\mathbf{D}=\mathbf{A}^{-1}-\mathbf{W}^{o}, \quad \mathbf{D}^{o}=\mathbf{A}^{-1} \mathbf{B}+\mathbf{L}^{o}$ where $\mathbf{D}, \mathbf{D}^{o}$ are the matrix and vector characterizing at step $\left[t_{j}, t_{j+1}\right]$ movement of the "car track" system.

Let us trace the progress of the entire problem solution for $j=0,1,2, \ldots$ On the step $\left[t_{j}, t_{j+1}\right]$ under the initial conditions of the problem at the time $t_{j}$ is determined using (14), the vector $\ddot{\mathbf{q}}_{k o j+1 / 2}^{o}$, then, using (13), (12), and (2), the fields of displacements, velocities, and reactions for the system $\left\{e_{h}, e_{h^{o}}^{\circ}\right\}$ in the moment $t_{j+1}$. Then the process is repeated.

\section{Numerical simulation results}

The step-by-step procedure (14) is implemented for a high-speed train from [6] when moving along the experimental track section. The system $\left\{e_{h}, e_{h^{o}}^{\circ}\right\}$ consists of a rail-beam on an elastic foundation with a length $\ell=108 \mathrm{~m}$ and a car simulated by a mechanical system with 10 degrees of freedom, consisting of rigid bodies with elastic-viscous constraints, describing the vertical dynamics of the car during high-speed movement with variable speed, including emergency. Path parameters, as in the first part of the article, for a path segment with the length $\ell=13 \mathrm{~m}$, when studying the dynamics of the system $\left\{e_{h}, e_{h^{o}}^{\circ}\right\}$ coincide, taking into account the energy dissipation in the system at $\gamma=6 \mathrm{kNs} / \mathrm{m}^{2} ; k=$ $30000 \mathrm{kN} / \mathrm{m}^{2} ; \mu_{1}=0$. The inertial and elastic-viscous parameters of the car are selected from [6]. To test the system $\left\{e_{h}, e_{h^{o}}^{\circ}\right.$ operation, an additional numerical experiment was carried out. The problem was considered for determining the support reactions of the car at $v=0$ and its support on a rigid base and the action of a moment suddenly applied to the body, equal to the inertial moment when the car is braking. As a result, Fig. $3 b$ shows the changes over time $t$ (c) dynamic reactions $R_{i}(\mathrm{kN})$ the first and fourth wheelsets. When the car is moving, its position on the track section is determined by the segment $s(t)$ (Fig. 4), and, accordingly, for the fourth wheel pair by the segment $s_{4}$. The system of equations (14) changed its order from 1 to 4 in the process of numerical implementation, when the car was moving at a speed $v_{0}=250 \mathrm{~km} / \mathrm{h}$ and from the beginning of the car braking at the moment when $s=0.5 \ell$ and $w=-2 \mathrm{~m} / \mathrm{s}^{2}$ [7]. The dynamic addition change $R(\mathrm{kN})$ of the fourth wheelset of the car to the static pressure of this wheelset equal to $P=170 \mathrm{kN}$ depending on $s(t)$ is shown in Fig. $5 a$. Fig. $5(b, c)$ shows, respectively, when the car is moving, depending on $t$ and $s(t)$ (Fig. 4) the vertical displacements: fourth wheel $q_{4}$ [m] (line 1 ) and the middle of the rail-beam on the elastic foundation $q_{1 b}$ (line 2) (Fig. 5b) and the center of mass of the car body $q[\mathrm{~m}]$ (line 1 ) and the corner point of the car $A$ (Fig. 4 (line 2) (Fig. 5c)). Integration step $\Delta t_{j}$ by $n=580$ in (4) was chosen equal to $\Delta t_{j}=0.00072 \mathrm{~s}$, with the number of steps $N=2160$ to implement the procedure (11) - (14). It should be 
noted that the expected coincidence of the graph ordinates in Fig. $5 b$, corresponding to the moment when the fourth wheel of the car reaches the middle of the experimental section of the rail at $\mathrm{s}=75.4 \mathrm{~m}$. The process emergence of unloading the fourth wheelset (in the form of a drop in the value of the dynamic additive $R$ to the static reaction of the wheel (see Fig. $4 a$ ), which coincides in magnitude with the result shown in Fig. 3b, and caused by the action during braking of the inertial pair $M^{(и \mu)}$ (Fig. 4), at the moment the car starts braking should also be noted.

\section{Conclusion}

The proposed method makes it possible to investigate the action of a movable inertial load with a variable movement speed on the rods on an elastic foundation, under various boundary conditions, while applying the corresponding fundamental functions using the step-by-step procedure proposed in [4]. Applied to the problems of railway transport, the method allows to investigate the interaction process (Fig. 5c) in the system "car - track" at different speeds of the train in the modes of the beginning of braking or its acceleration, at any position along the track section and the possible presence of various irregularities $[5,8]$.

\section{References}

1. L. Fryba, Vibration of solids and structures under moving loads (Academia, Prague, 1972).

2. S.S. Kokhmanyuk, A.P. Filippov, Construction mechanics and design of structures 2, 36-39 (1967).

3. B.G. Korenev, I.M. Rabinovich, Dynamic calculation of buildings and structures, Designer handbook, Edition 2 (Stroyizdat, Moscow, 1984).

4. I.I. Ivanchenko, Prikl. Mechanics 24 (9), 109-118 (1988).

5. I.I. Ivanchenko, RAS. MTT 4, 158-177 (2005).

6. A.Ya. Kogan, A.A. Lvov, M.A. Levinzon, Railway Research Institute (JSC VNIIZhT). 3, 10-14 (1991).

7. R.U. Naumenko, I.Yu. Khizha, E.G. Bogomaz, Bulletin of the Dnipropetrovsk National University of Railway Transport 29, 44-48 (2009).

8. I.I. Ivanchenko, Dynamics of transport structures (high-speed mobile, seismic and shock loads) (Publishing house Nauka, Moscow, 2011).

9. I.I. Ivanchenko, IOP Conf. Series: Materials Science and Engineering. Sci. Eng. 913, 032062 (2020). 